\title{
INFRARED BEHAVIOR OF THE RESPONSE OF STRONGLY INTERACTING BOSE SYSTEMS
}

\author{
A. Chumachenko ${ }^{1}$, S. Vilchynskyy ${ }^{1}$, M. Weyrauch ${ }^{2}$ \\ ${ }^{1}$ National Taras Shevchenko University, Kyiv, Ukraine \\ ${ }^{2}$ Physikalisch-Technische Bundesanstalt, \\ D-38116 Braunschweig, Federal Republic of Germany
}

(Received December 18, 2006; received in final form January 10, 2007)

\begin{abstract}
We discuss field-theoretical foundations of Landau's quasi-particle concept for strongly interacting Bosons. The historical development starting from Belyaev's field theory up to modern renormalization group theory is briefly reviewed with a particular emphasis on the problem of infrared divergences. It is shown in detail that the correlation functions obtained in Popov's functional integral approach based on a hydrodynamic action agrees precisely with recent renormalization group analysis. The phonon decay is also discussed.
\end{abstract}

Key words: infrared divergence, hydrodynamical approach, renormalization.

PACS number(s): 67.40.-w, 11.30.Qc, 11.10.Gh

\section{INTRODUCTION}

Landau's phenomenological theory for strongly interacting Bose systems [1,2] asserts that the low lying excitations may be conveniently represented by noninteracting quasi-particles. This bold assumption describes many experimental observations successfully. At long wavelength, the quasi-particles are phonons with a linear relation $\omega=c|\mathbf{q}|$ between energy $\omega$ and momentum $\mathbf{q}$ and the (first) sound velocity $c$ (we use units such that $\left.\hbar=k_{\mathrm{B}}=1\right)$. For superfluid ${ }^{4} \mathrm{He}$ at larger momenta, the quasi-particle spectrum is given by the well-known maxon-roton dispersion relation.

How can this extremely successful phenomenological description be understood from the underlying field theory? In this paper we focus on this question with a particular emphasis on the phonon region. In fact, a fieldtheoretical understanding of the response of interacting Bose systems at small energies and momenta is surprisingly difficult: The first important steps towards a field theory for strongly interacting Bosons were made in the 1950's by Bogoliubov [3], Belyaev [4,5], Hugenholtz and Pines [6] and later by Gavoret and Nozières [7].

In order to take into account Bose condensation, which is a macroscopic occupation of the zero momentum component of the Bose field, Bogoliubov [3] suggested treating the corresponding field operator $\hat{a}_{0}$ as a c-number

$$
\hat{a}_{0} \rightarrow\left\langle\hat{a}_{0}\right\rangle=\sqrt{n_{0}}
$$

with $n_{0}$ the condensate density. He was then able to approximately diagonalize the many-body Hamiltonian for a weakly interacting gas of Bosons and obtained its quasiparticle excitation spectrum

$\omega(\mathbf{q})=\left[\frac{\mathbf{q}^{2}}{2 m}\left(\frac{\mathbf{q}^{2}}{2 m}+2 n_{0} V(\mathbf{q})\right)\right]^{1 / 2} \rightarrow c|\mathbf{q}|$ for $\mathbf{q} \rightarrow 0$ with $c=\sqrt{n_{0} V(0) / m}$. This spectrum is acoustic for small momenta. In order to obtain a stable spectrum, the Bose particle interaction $V(\mathbf{q})$ must fulfill the condition $q^{2}>4 m n_{0} V(\mathbf{q})$ with $m$ the Boson mass.

While Bogoliubov's theory was a great step forward in the understanding of the low lying spectrum of interacting Bosons, the theory is certainly not applicable to strongly interacting systems like superfluid ${ }^{4} \mathrm{He}$. However, the theory paved the way for many important developments, e.g., the concept of symmetry breaking, which plays such a prominent role in modern theoretical physics, is implicitly introduced through Eq. (1). The symmetry broken by Eq. (1) is the gauge symmetry, and as a consequence, the particle number is not a conserved quantity. As we shall see, the gauge symmetry breaking plays a very important role in the understanding of the infrared structure of the Bose system. A very lucid discussion of the concept of symmetry breaking and its implications is given by Anderson in Ref. [9].

The many-body field theory for Bose systems was first developed by Belyaev [4]. Since the Bose condensate acts as a particle reservoir from which particles can be created or into which particles may be lost, it was necessary to introduce "anomalous" Green's functions in order to describe such processes. Therefore, the Dyson equation for the Green's functions

$$
G=G_{0}+G_{0} \Sigma G
$$

is turned into a $2 \times 2$ matrix equation. The diagonal elements in this equation correspond to the conventional Green's functions, and the off-diagonal elements in $G$ and $\Sigma$ are the anomalous Green's functions and self-energies, respectively.

The resulting set of equations may be easily solved, e.g., for $G_{11}$ 


$$
G_{11}(\omega, \mathbf{q})=\frac{\omega+\mathbf{q}^{2} / 2 m-\mu+\Sigma_{22}}{\left[\omega-\frac{1}{2}\left(\Sigma_{11}-\Sigma_{22}\right)\right]^{2}-\left[\mathbf{q}^{2} / 2 m-\mu+\frac{1}{2}\left(\Sigma_{11}+\Sigma_{22}\right)\right]^{2}+\Sigma_{21}^{2}} .
$$

Here we have used a free propagator $\left(G_{0}\right)_{11}^{-1}=\omega+\mathbf{q}^{2} / 2 m-\mu$ with $\mu$ being the chemical potential. In order to determine the spectrum, we must calculate the self energies. If one does this in the lowest order of perturbation theory, Bogoliubov's result (2) is reproduced exactly (see e.g. Ref. [10]). Furthermore, a finite value for $\Sigma_{12}(0)$ is obtained. However, already in the second order of perturbation theory one obtains infrared divergent terms. An example is shown in Fig. 1.

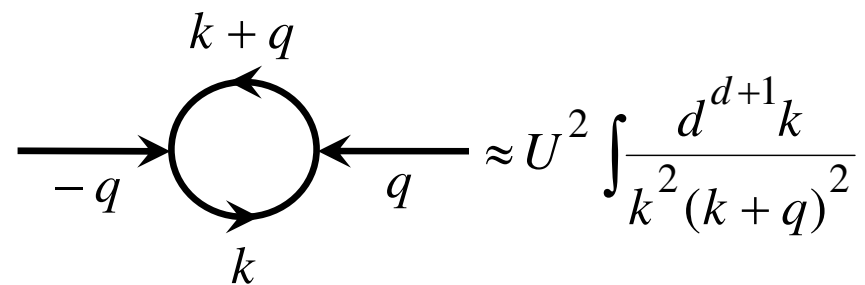

Fig. 1. Perturbative contribution to the anomalous Green's function $G_{12}$. This diagram diverges in 3 dimensions in the infrared. It is calculated at $T=0$ introducing a 4 -momentum $q=(\omega / c, \mathbf{q})$.

The handling of these divergences in a satisfactory manner is necessary in order to understand the infrared response of a Bose system. It requires a suitable renormalization procedure. Unfortunately, this proves to be a rather difficult problem.

Belyaev's theory was reanalyzed and extended by Hugenholtz and Pines [6]. Using the gauge invariance arguments and a careful analysis of the perturbation series they showed that the quasi-particle spectrum is gapless. In particular, they obtained that

$$
\Sigma_{11}(0)-\Sigma_{12}(0)=\mu
$$

(Hugenholtz-Pines theorem). Inserting this theorem into Eq. (4), it is easily established that the spectrum is gapless. It is now tempting to obtain the infrared behavior of the Green's functions directly from the Belyaev equations (4). Assuming the self-energy parts $\Sigma_{11}$ and $\Sigma_{12}$ to be analytic at small momenta one obtains

$$
G_{11}(\omega, \mathbf{q})=\frac{\Sigma_{11}(0)-\mu}{B\left(\omega^{2}-c^{2} \mathbf{q}^{2}\right)}=-\frac{\Sigma_{12}(0)}{B\left(\omega^{2}-c^{2} \mathbf{q}^{2}\right)}
$$

where $B$ and $c$ are constants involving derivatives of the self-energies at $q=0$. Here, one also finds that $\Sigma_{12}(0) \neq 0$. The argument just presented is given in more detail in Ref. [10], and it indeed leads to a linear spectrum. However, it assumes analyticity of the self-energies and Green's functions at zero momentum, which appears to be erroneous as will be discussed shortly.

The field theory for Bose systems as developed by Belyaev and Hugenholtz and Pines was further extended by Gavoret and Nozières [7]. They established the phonon character of the spectrum up to all orders in perturbation theory. Furthermore, they successfully related the the sound parameter of the field theoretical propagator with the macroscopic sound velocity $c$ given by $c^{2}=d p / d \rho$ where $p$ is the pressure and $\rho$ the mass density of the Bose system. The theory of Gavoret and Nozières effectively sums up perturbation theory to infinite order, but it does not solve the problem with infrared divergences. It yields an anomalous self-energy $\Sigma_{12}(0) \neq 0$.

The first satisfactory attempt to handle the infrared divergences of the Bosonic field theory was undertaken by A. A. Nepomnyashchii and Yu. A. Nepomnyashchii [11] (NN). Their calculations are rather involved and entail a partial summation of the perturbation series. If this resummation of diagrams is done correctly then infrared divergences disappear from the theory. As an important consequence of this diagrammatic analysis one obtains that the long wavelength behavior of the anomalous self-energy is actually nonanalytic at $q=0$,

$$
\Sigma_{12}(\omega \rightarrow 0, \mathbf{q} \rightarrow 0) \sim \frac{1}{\ln \left(q_{0} / q\right)}
$$

Here, $q=(\omega / c, \mathbf{q})$ and $1 / q_{0}$ is the length of the order of the interparticle distance. Eq. (7) is a very important result, which makes Green's functions $G_{11}$ and $G_{12}$ also behave non-analytically at $(\omega, \mathbf{q})=0$. Obviously, Eq. (7) leads to $\Sigma_{12}(0)=0$, which contradicts Eq. (6). NN confirmed that the spectrum remains acoustic despite the non-analytic behavior of the correlation functions.

The method applied by NN in order to remove the infrared divergences from the Bosonic field theory is certainly not very transparent. It would be desirable to be able to construct a perturbation theory where infrared divergences are eliminated from the outset. Such a perturbation expansion has been suggested by Popov $[12,13]$ starting from a functional integral approach. This approach yields the same perturbation expansion as the conventional field theoretical approach but suggests a more convenient method to eliminate infrared divergences. Popov's method is based on the separation of the Bosonic fields into "fast" and "slow" components with respect to a certain momentum $q_{0}$. Integrating out the "fast" fields, Popov was able to construct an effective action for the "slow" fields only. Representing the "slow" 


\section{A. CHUMACHENKO, S. VILCHYNSKYY, M. WEYRAUCH}

fields by their amplitude and phase one obtains an effective hydrodynamic action. The diagram technique obtained from this action is free of infrared divergences. It is then straightforward to calculate the infrared structure of the various correlation functions. In the following section we will present this development in some detail. Popov and Serednyakov [14] were able to obtain Eq. (7), which was first derived by NN, from the effective hydrodynamic action. It implies that the Green's functions obtained in Bogolubov's theory are not correct despite the fact that an acoustic spectrum is obtained.

While the method employed by Popov entails essential ideas of modern renormalization group theory, it still contains a number of phenomenological elements. In particular a sharp separation of the fields into "slow" and "fast" components at the given momentum $q_{0}$ appears to be somewhat artificial and, furthermore, the parameter $q_{0}$ is not really well defined. A full-fledged renormalization group analysis of infrared behavior of the Green's functions of a Bose system was undertaken only recently by Pistolesi et al. $[15,16]$. Within this theory it was explicitly shown that the effective hydrodynamic action proposed by Popov is indeed the correct infrared fixed point of the renormalization group flow which starts at the "bare" action of strongly interacting Bosons. To show this, Pistolesi et al. write down a general form of the action in terms of "running" couplings and find that all the couplings that are present in the "bare" interaction but not in the hydrodynamic action flow to zero or are irrelevant. This beautiful analysis confirms all the results obtained in the NN and Popov approaches.

In order to expose the effects of the broken gauge symmetry on the Green's functions, the Bose fields are separated into longitudinal and transverse components in Ref. [15]. The gauge symmetry is broken in the longitudinal component only. Using this formulation it is particularly easy to set up Ward identities, which relate various vertices to each other. We develop Popov's theory here using an analogous separation of the fields into longitudinal and transverse components. The Ward identities are then used in order to obtain the vertices for the calculation of the density-density and current-current correlation functions.
The vanishing of the anomalous self-energy $\Sigma_{12}(0)$ at zero momentum has a definite physical origin and is not just a peculiar mathematical result. In the framework of broken symmetry, it is consistent with the general picture proposed by Patashinskii and Pokrovskii [17] where divergences, which arise in transverse correlation functions connected with a Goldstone mode (zero mass phonon), drive a divergence in the longitudinal propagators due to the continuously broken symmetry. From this point of view, the divergence of Green's functions at zero momentum due to the vanishing of $\Sigma_{12}$ is an immediate consequence of the Goldstone mode.

In this paper, we show explicitly that the results of the renormalization group analysis presented in Ref. [15] and the Popov approach coincide. In order to do this, we need to consider slight modifications to Popov's original work. Explicit formulas for the correlation functions will be presented, and various applications will be discussed.

Besides the field-theoretical methods discussed in this paper, there are various other methods that attempt to provide a microscopic basis for Landau's quasi-particle concept. We will not enter into a comparison of these methods here, but just mention a few of them for completeness: The hydrodynamic formulation by Hohenberg and Martin [8] describes the infrared response without the problem of spurious infrared divergences, but it does not obtain the important result that $\Sigma_{12}(0)=0$. A very good quantitative description of the response of superfluid ${ }^{4} \mathrm{He}$ at long and intermediate wavelength is obtained using numerical quantum Monte-Carlo. For a review see Ceperley Ref. [18]. Finally, a very complete picture of the excitations of Bose systems is obtained using "correlated bases functions", a method based on a seminal work by Feynman [19] and Feenberg [20], which is also at the root of a recent theory of superfluid ${ }^{4} \mathrm{He}$ by I. O. Vakarchuk [21].

\section{POPOV'S HYDRODYNAMIC APPROACH}

We consider a system of strongly interacting neutral bosons. The associated gauge invariant action is given by $[12,15]$

$$
\begin{aligned}
& S\left[\psi^{*}, \psi, \mu, \mathbf{A}, J^{*}, J\right]=\int_{0}^{\beta} \mathrm{d} \tau \int \mathrm{d}^{d} r\left\{\psi^{*}(x)\left[\partial_{\tau}+\mu(x)\right] \psi(x)\right. \\
& \left.-\frac{1}{2 m}|(\nabla-i \mathbf{A}) \psi(x)|^{2}-\frac{U}{2}|\psi(x)|^{4}+\psi(x) J^{*}(x)+\psi^{*}(x) J(x)\right\} .
\end{aligned}
$$

In this expression, $x=(\tau, \mathbf{r})$ is a vector with $d+1$ dimensions. The imaginary time $\tau$ ranges from 0 to the inverse temperature $\beta$. The bosonic field $\psi(x)$ is periodic in the imaginary time, and $U$ is a short-range two-body interaction potential. The external sources $J(x), \mu(x) \equiv A_{0}(x)$ and $\mathbf{A}(x)$ serve to generate the various connected correlation functions by a functional differentiation of the free energy functional

$$
F\left[\mu, \mathbf{A}, J^{*}, J\right]=\beta \ln \left\{\int \mathrm{D} \psi^{*} \mathrm{D} \psi \exp \left(-S\left[\psi^{*}, \psi, \mu, \mathbf{A}, J^{*}, J\right]\right)\right\} .
$$

For details we refer e.g. to Ref. [22]. 
The perturbation theory based on action (8) is plagued by infrared divergent integrals. These divergences must be removed by a suitable renormalization procedure as was done in Ref. [15, 16]. Here instead, we will use Popov's hydrodynamic approach [12] and show that it yields exactly the same correlation functions as the renormalization group approach. As was already alluded to in the introduction, Popov's hydrodynamic approach is based on a separation of the Fourier components of the Bose field $\psi(\mathbf{x})$ into "fast" $\psi_{f}(x)$ and "slow" $\psi_{s}(x)$ components with respect to a fixed momentum $q_{0}$. The "fast" component of the field is integrated out, and one obtains an effective (renormalized) action $S_{h}\left[\psi_{s}^{*}, \psi_{s}\right]$ which depends on the "slow" fields only. This effective action will be called "hydrodynamic" in the following. The perturbation theory based on this effective action is free of infrared divergences (in three dimensions) as shown in Ref. [12] and easily confirmed by power counting.

It turns out to be convenient to express the "slow" fields in terms of the modulus $n(x)$ and the phase $\varphi(x)$

$$
\psi_{s}(x)=\sqrt{n(x)} e^{i \varphi(x)}, \quad \psi_{s}^{*}(x)=\sqrt{n(x)} e^{-i \varphi(x)}
$$

since the Fourier transform of the effective action takes a simple form in terms of these variables [12],

$$
\begin{aligned}
& S_{h}[\pi, \varphi]=\frac{1}{2 \beta \Omega} \sum_{q}\left\{-\left(\frac{p_{\mu}}{m} \mathbf{q}^{2}+p_{\mu \mu} \omega_{n}^{2}\right) \varphi(q) \varphi(-q)\right. \\
& \left.-2 p_{\mu n_{0}} \omega_{n} \varphi(q) \pi(-q)+\left(p_{n_{0} n_{0}}-\frac{\mathbf{q}^{2}}{4 m n_{0}}\right) \pi(q) \pi(-q)\right\} \\
& +\frac{1}{(\beta \Omega)^{\frac{3}{2}}} \sum_{q_{1}+q_{2}+q_{3}=0} \frac{\mathbf{q}_{1} \cdot \mathbf{q}_{2}}{2 m} \varphi\left(q_{1}\right) \varphi\left(q_{2}\right) \pi\left(q_{3}\right)
\end{aligned}
$$

Here the variable $\pi$ is defined by $\pi(x)=n(x)-n_{0}\left(q_{0}\right)$, where $n_{0}\left(q_{0}\right)=n_{0}$ is the "bare" condensate, which is determined from the condition $p_{n_{0}}=0$, and $\Omega$ is a normalization volume. Furthermore, we introduce a 4 momentum notation by $q=\left(\omega_{n} / c, \mathbf{q}\right)$, where $c$ is the speed of sound to be discussed below and the BoseMatsubara frequency $\omega_{n}=2 \pi n \beta^{-1}$ with $n$ an integer. For simplicity the gauge fields $\mathbf{A}(x)$ and $\mu(x)$ are set to zero and the chemical potential $\mu$, respectively.

The coefficients $p_{\mu}, p_{\mu \mu}, p_{\mu n_{0}}$ and $p_{n_{0} n_{0}}$ are thermodynamic derivatives of the pressure $p\left(\mu, n_{0}\right)=$ $S_{h}\left(\mu, n_{0}\right) / \beta \Omega$ with respect to the chemical potential.
The quadratic part of the action determines the "unperturbed" Green's functions $G_{0}$. The cubic term (last term in Eq. (11)) will be treated as a perturbation. This part gives rise to the quasi-particle decay discussed in Section II C. The average of a quantity $O$ with respect to the quadratic part of the action will be denoted by $\langle O\rangle_{0}$.

The unperturbed correlation functions $g_{\pi \pi}(q)=$ $\langle\pi(q) \pi(-q)\rangle_{0}, \quad g_{\varphi \varphi}(q)=\langle\varphi(q) \varphi(-q)\rangle_{0}, \quad g_{\pi \varphi}(q)=$ $\langle\pi(q) \varphi(-q)\rangle_{0}=-g_{\varphi \pi}(q)$ are easily calculated from Eq. (11)

$$
\begin{aligned}
G_{0}(q) & =\left(\begin{array}{cc}
g_{\pi \pi}(q) & g_{\pi \varphi}(q) \\
g_{\varphi \pi}(q) & g_{\varphi \varphi}(q)
\end{array}\right) \\
& =-\left(\begin{array}{cc}
\frac{p_{\mu}}{m} \mathbf{q}^{2}+p_{\mu \mu} \omega_{n}^{2} & p_{\mu n_{0}} \omega_{n} \\
-p_{\mu n_{0}} \omega_{n} & -p_{n_{0} n_{0}}+\frac{\mathbf{q}^{2}}{4 m n_{0}}
\end{array}\right) \frac{1}{D(q)}
\end{aligned}
$$

with

$$
\begin{aligned}
D(q) & =\left(\frac{\mathbf{q}^{2}}{2 m}\right)^{2} \frac{p_{\mu}}{n_{0}}+\frac{p_{\mu \mu}}{4 m n_{0}} \mathbf{q}^{2} \omega_{n}^{2}-\left(p_{n_{0} n_{0}} p_{\mu \mu}-p_{n_{0} \mu}^{2}\right) \\
& \times\left(\omega_{n}^{2}+\frac{1}{m} \frac{p_{n_{0} n_{0}} p_{\mu}}{p_{n_{0} n_{0}} p_{\mu \mu}-p_{n_{0} \mu}^{2}} \mathbf{q}^{2}\right) .
\end{aligned}
$$

The first two terms in $D(q)$ represent a non-linear correction to the spectrum and can be neglected in the small $|\mathbf{q}|, \omega_{n}$ limit. The ratio

$$
\frac{1}{m} \frac{p_{n_{0} n_{0}} p_{\mu}}{p_{n_{0} n_{0}} p_{\mu \mu}-p_{\mu n_{0}}^{2}}=\frac{n}{m} \frac{d n}{d \mu}=\frac{d p}{d \rho}=c^{2}
$$

can be identified with the square of macroscopic sound velocity $[7,15]$. Here, we used that $p_{\mu}=n$ with $n$ the total density of the system. The quantity [15]

$$
\frac{p_{\mu n_{0}}}{p_{n_{0} n_{0}}}=-\frac{\mathrm{d} n_{0}}{\mathrm{~d} \mu}
$$

is known as the "condensate" compressibility. Furthermore, it is easy to show that

$$
\frac{p_{\mu \mu}}{p_{n_{0} n_{0}}}=\frac{n}{m c^{2}} \frac{1}{p_{n_{0} n_{0}}}+\left(\frac{\mathrm{d} n_{0}}{\mathrm{~d} \mu}\right)^{2} .
$$

Using these relations the matrix of correlation functions given in Eq. (12) may be written as

$$
G_{0}(q)=-\left(\begin{array}{cc}
-\left(\frac{\mathrm{d} n_{0}}{\mathrm{~d} \mu}\right)^{2} \omega_{n}^{2} & \frac{\mathrm{d} n_{0}}{\mathrm{~d} \mu} \omega_{n} \\
-\frac{\mathrm{d} n_{0}}{\mathrm{~d} \mu} \omega_{n} & 1
\end{array}\right) \frac{m}{n} \frac{c^{2}}{\omega_{n}^{2}+c^{2} \mathbf{q}^{2}}+\left(\begin{array}{cc}
\frac{1}{p_{n_{0} n_{0}}} & 0 \\
0 & \frac{1}{8 n n_{0}} \frac{1}{p_{n_{0} n_{0}}}
\end{array}\right)
$$

Obviously, $g_{\pi \pi}$ is finite at small $q$, while the other correlation functions are infrared divergent. This is due to the degeneracy of the Bose system in the $\varphi$ direction due to a Goldstone (massless) mode. This Goldstone mode is related to a spontaneous gauge symmetry breaking. The last term in Eq. (17) may be neglected at small $q$ and $\omega$. Our result agrees with Popov's [12] in the low density limit, in which the various coefficients are given by 


\section{A. CHUMACHENKO, S. VILCHYNSKYY, M. WEYRAUCH}

$$
p_{\mu}=n \approx n_{0}, \quad p_{\mu \mu}=0, \quad p_{\mu n_{0}}=1, \quad p_{n_{0} n_{0}}=-\frac{m c^{2}}{n_{0}}
$$

In the following subsections we use $G_{0}(q)$ to obtain the single particle propagators, associated vertices, densities and density-density correlation functions.

\section{A. Single particle propagator and vertex functions}

We now calculate Green's functions $G_{11}(x, y)$ and $G_{12}(x, y)$ which are defined in terms of the fields $\psi_{s}(x)$. The index $s$ will be omitted in the following. As was already alluded to in the introduction, it is advantageous to separate the fields into longitudinal and transverse components $\psi_{l}$ and $\psi_{t}$

$$
\begin{aligned}
& \psi(x)=\psi_{l}(x)+i \psi_{t}(x), \quad \psi_{l}(x)=\sqrt{n(x)} \cos \varphi(x), \\
& \psi_{t}(x)=\sqrt{n(x)} \sin \varphi(x),
\end{aligned}
$$

since the gauge symmetry is broken in the longitudinal component only. Green's functions are then written as

$$
G_{i j}(x, y)=\left\langle\psi_{i}(x) \psi_{j}(y)\right\rangle_{0}-\left\langle\psi_{i}(x)\right\rangle_{0}\left\langle\psi_{j}(x)\right\rangle_{0}
$$

for $\{i, j\} \in\{l, t\}$. Now we expand $\psi_{l}(x)$ and $\psi_{t}(x)$ into a power series of $\pi(x)=n(x)-n_{0}$ and $\varphi(x)$,

$$
\begin{aligned}
& \psi_{l}(x)=\sqrt{n_{0}}+\frac{1}{2 \sqrt{n_{0}}} \pi(x)-\frac{1}{2} \sqrt{n_{0}} \varphi^{2}(x)+\ldots, \\
& \psi_{t}(x)=\sqrt{n_{0}} \varphi(x)+\frac{1}{2 \sqrt{n_{0}}} \pi(x) \varphi(x)+\ldots,
\end{aligned}
$$

and use that $\left\langle\psi_{l}(x)\right\rangle_{0}=\sqrt{n_{0}}$ and $\left\langle\psi_{t}(x)\right\rangle_{0}=0$. At small momenta only the lowest powers in $\pi$ and $\varphi$ contribute. Assuming translational invariance, we obtain for the Fourier transform of Green's functions at small $q$

$$
\begin{aligned}
G_{l l}(q \rightarrow 0) & =\frac{1}{4 n_{0}} g_{\pi \pi}(q) \\
& -\frac{n_{0}}{2} \int_{q^{2}<q_{0}^{2}} \frac{\mathrm{d}^{d+1} p}{(2 \pi)^{d+1}} g_{\varphi \varphi}(p) g_{\varphi \varphi}(q-p), \\
G_{l t}(q \rightarrow 0) & =\frac{1}{2} g_{\pi \varphi}(q), \\
G_{t t}(q \rightarrow 0) & =n_{0} g_{\varphi \varphi}(q),
\end{aligned}
$$

where we used that at $T=0$ the sum over the Matsubara frequencies turns into an integral according to

$$
\frac{1}{\beta} \sum_{n=-\infty}^{\infty}=\int \frac{\mathrm{d} \omega}{2 \pi} .
$$

Inserting the correlation functions Eq. (17) into Eqs. (22) one finds for $d=3$ the important results

$$
\begin{aligned}
G_{l l}(q \rightarrow 0) & =\frac{m}{4 n_{0} n}\left(\frac{d n_{0}}{d \mu}\right)^{2} \frac{c^{2} \omega^{2}}{\omega^{2}+c^{2} \mathbf{q}^{2}} \\
& -\frac{n_{0}}{2} \frac{m^{2} c}{n^{2}} \frac{1}{8 \pi^{2}} \ln \frac{k_{0}}{q}
\end{aligned}
$$

$$
\begin{aligned}
& G_{l t}(q \rightarrow 0)=-\frac{m}{2 n} \frac{d n_{0}}{d \mu} \frac{c^{2} \omega}{\omega^{2}+c^{2} \mathbf{q}^{2}}=G_{t l} \\
& G_{t t}(q \rightarrow 0)=-\frac{m n_{0}}{n} \frac{c^{2}}{\omega^{2}+c^{2} \mathbf{q}^{2}} .
\end{aligned}
$$

We note that these results exactly agree with those obtained in the renormalization group approach presented in Ref. [15] (see their Eqs. (4.18), (4.19), and (4.27)). All correlation functions are infrared divergent at $(\omega, \mathbf{q})=0$. The longitudinal correlation function, which one would expect to be finite in the infrared limit, acquires a divergence due to the "coupling" to the phase fluctuations. In $3 \mathrm{D}$ this divergence is logarithmic. It has been identified for the first time by NN Ref. [11] and was later confirmed by Popov and Serednyakov in Ref. [14].

The normal and anomalous Green's functions, $G_{11}$ and $G_{12}$, are now easily calculated

$$
\begin{aligned}
G_{1 j}(q \rightarrow 0) & =\mp \frac{m n_{0}}{n} \frac{c^{2}}{\omega^{2}+c^{2} \mathbf{q}^{2}} \\
& -\frac{n_{0}}{2} \frac{m^{2} c}{n^{2}} \frac{1}{8 \pi^{2}} \ln \frac{k_{0}}{q}
\end{aligned}
$$

with the "-" $\operatorname{sign}$ for $j=1$ and the "+" $\operatorname{sign}$ for $j=2$, and we kept only the divergent terms at small momenta. The above result corresponds exactly to the one given by Nepomnyashchii Ref. [23] (Eq. (7)). In Ref. [7], Eq. (4.15), the logarithmcally diverging term is missing.

An important quantity calculated from Greens's function is the momentum distribution

$$
n(\mathbf{q}, \beta)=-\frac{1}{\beta} \sum_{m=-\infty}^{\infty} G_{11}(i \omega, \mathbf{q}) .
$$

For $T=0$ one immediately obtains the well-known result [24] from the first term in Eq. (25)

$$
n(\mathbf{q} \rightarrow 0)=\frac{m n_{0} c}{2 n} \frac{1}{|\mathbf{q}|},
$$

where we used $\operatorname{coth}(x) \approx 1$ for large $x$. The contribution of the second term is constant.

We will now briefly discuss the results for finite temperatures: In the classical regime $T>c|\mathbf{q}|$ we replace Eqs. (22) by

$$
\begin{aligned}
& G_{l l}(0, \mathbf{q} \rightarrow 0)=-\frac{n_{0}}{2 \beta} \\
& \times \sum_{n=-\infty}^{\infty} \int \frac{\mathrm{d}^{d} p}{(2 \pi)^{d}} g_{\varphi \varphi}\left(\omega_{n}, \mathbf{p}\right) g_{\varphi \varphi}\left(\omega_{n}, \mathbf{q}-\mathbf{p}\right) \\
& G_{l t}(0, \mathbf{q} \rightarrow 0)=0 \\
& G_{t t}(0, \mathbf{q} \rightarrow 0)=-\frac{n_{0} m}{n} \frac{1}{\mathbf{q}^{2}} .
\end{aligned}
$$

Evaluating the Matsubara sum and the integral for $d=3$ one finds

$$
G_{l l}(0, \mathbf{q} \rightarrow 0)=-\frac{1}{16} \frac{1}{\beta|\mathbf{q}|} \frac{m^{2} n_{0}}{n^{2}},
$$


and

$$
G_{1 j}(0, \mathbf{q})=\mp \frac{n_{0} m}{n} \frac{1}{\mathbf{q}^{2}}-\frac{1}{16} \frac{1}{\beta|\mathbf{q}|} \frac{m^{2} n_{0}}{n^{2}},
$$

with the "-" sign for $j=1$ and the "+" sign for $j=2$. From this result we find the momentum distribution

$$
n(\mathbf{q} \rightarrow 0, \beta)=\frac{1}{\beta} \frac{m n_{0}}{n} \frac{1}{\mathbf{q}^{2}}+\frac{1}{16 \beta^{2}} \frac{m^{2} n_{0}}{n^{2}} \frac{1}{|\mathbf{q}|} .
$$

The last term originates from the $g_{\varphi \varphi} g_{\varphi \varphi}$ correction to $G_{l l}$. This term may be identified experimentally at very small q.

Finally we calculate the vertex functions. The vertices are generated from the Legendre transform of the free energy Eq. (9) (Gibb's free energy) $\Gamma\left[\phi_{i}, A_{\nu}\right]$ with respect to the sources $J_{i}$, i.e. $\delta \Gamma / \delta \phi_{i}(x)=J_{i}(x)$ and $i \in\{l, t\}$. For further technical details see Refs. [15,22]. For the various functional derivatives we use the notation introduced in Ref. [15], i.e. $\Gamma_{i_{1} \ldots i_{n} ; \nu_{1}, \ldots \nu_{n}}$. The indices $i_{k}$ to correspond a functional derivative with respect to $\phi_{i_{k}}$ and the indices $\nu_{k}$ to functional derivatives with respect to $A_{\nu_{k}}$. Specifically one finds

$$
\begin{aligned}
& \Gamma_{l l}(q \rightarrow 0)=-16 \pi^{2} \frac{n^{2}}{n_{0}} \frac{1}{m^{2} c} \frac{1}{\ln \left(q_{0} / q\right)}, \\
& \Gamma_{l t}(q \rightarrow 0)=8 \pi^{2} \frac{n^{2}}{n_{0}^{2}} \frac{1}{m^{2} c} \frac{d n_{0}}{d \mu} \frac{\omega}{\ln \left(q_{0} / q\right)}=-\Gamma_{t l}, \\
& \Gamma_{t t}(q \rightarrow 0)=-\frac{n}{n_{0}} \frac{1}{m c^{2}}\left(\omega^{2}+c^{2} \mathbf{q}^{2}\right),
\end{aligned}
$$

from which the standard vertices and the self-energy $\Sigma_{12}(0)$ are derived,

$$
\begin{aligned}
\Gamma_{11}(q \rightarrow 0) & =\Gamma_{12}(q \rightarrow 0)=\Sigma_{12}(q \rightarrow 0) \\
& =-4 \pi^{2} \frac{n^{2}}{n_{0}} \frac{1}{m^{2} c} \frac{1}{\ln \left(k_{0} / q\right)} .
\end{aligned}
$$

Obviously, $\Sigma_{12}$ goes to zero for $q \rightarrow 0$ with an infinite slope at $\mathbf{q}=0$. Analogous results are easily derived for $T>c|\mathbf{q}|$.

\section{B. Two-particle correlation functions}

We now calculate the density-density and currentdensity response using

$$
G_{; \alpha \beta}(q)=\Gamma_{; \alpha \beta}(q)-\sum_{i, j \in l, t} \Gamma_{i ; \alpha}(-q) G_{i j}(q) \Gamma_{j ; \beta}(q) .
$$

Here we employ the notation defined in the previous subsection for the vertices and apply it in an analogous way for Green's functions. Green's functions are functional derivatives of the free energy Eq. (9), e.g. $G_{; \alpha \beta}$ represents a second order functional derivative of the free energy with respect to $A_{\alpha}(x)$ and $A_{\beta}(x)$. For $\alpha=\beta=0$ one obtains the density-density correlation function.

Using the Ward identities (see Ref. [15]) which follow from the gauge invariance of the action (8) and defining $q_{\nu}=(i \omega, \mathbf{q})$

$$
\begin{aligned}
& \Gamma_{t l}(q) \sqrt{n_{0}}-i q_{\nu} \Gamma_{l ; \nu}(-q)=0 \\
& \Gamma_{t t}(q) \sqrt{n_{0}}-i q_{\nu} \Gamma_{l ; \nu}(-q)=0 \\
& \Gamma_{t ; 0}(q) \sqrt{n_{0}}+i q_{\nu} \Gamma_{; \nu \mu}(-q)=0
\end{aligned}
$$

we are are able to find vertices,

$$
\begin{aligned}
\Gamma_{t ; 0}(\omega \rightarrow 0, \mathbf{q}=0) & =-\frac{n}{\sqrt{n_{0}}} \frac{\omega}{m c^{2}}, \\
\Gamma_{l ; 0}(\omega \rightarrow 0, \mathbf{q}=0) & =0, \\
\Gamma_{; 00}(\omega \rightarrow 0, \mathbf{q}=0) & =\frac{n}{m c^{2}}, \\
\Gamma_{l ; \alpha}(\omega=0, \mathbf{q} \rightarrow 0) & =0, \\
\Gamma_{t ; \alpha}(\omega=0, \mathbf{q} \rightarrow 0) & =-i \frac{n}{\sqrt{n_{0}}} \frac{\mathbf{q}_{\alpha}}{m}, \\
\Gamma_{; \alpha \beta}(\omega=0, \mathbf{q} \rightarrow 0) & =-\delta_{\alpha \beta} \frac{n}{m} .
\end{aligned}
$$

Using these vertices and propagators (24) one obtains for the density-density response

$$
\begin{aligned}
G_{; 00}(q) & =\frac{n}{m c^{2}}+\left(\frac{n}{\sqrt{n_{0}}} \frac{\omega}{m c^{2}}\right)^{2}\left(-\frac{m n_{0}}{n} \frac{c^{2}}{\omega^{2}+c^{2} \mathbf{q}^{2}}\right) \\
& =\frac{n}{m} \frac{\mathbf{q}^{2}}{\omega^{2}+c^{2} \mathbf{q}^{2}}
\end{aligned}
$$

which is the expected result. The density-density response is infrared finite. Analogously one finds for the current-current response

$$
\begin{aligned}
G_{; \alpha \beta}(q) & =-\frac{n}{m} \delta_{\alpha \beta}+\frac{n^{2}}{n_{0}} \frac{\mathbf{q}_{\alpha}}{m} \frac{\mathbf{q}_{\beta}}{m}\left(\frac{m n_{0}}{n} \frac{c^{2}}{\omega^{2}+c^{2} \mathbf{q}^{2}}\right) \\
& =-\frac{n}{m}\left(\delta_{\alpha \beta}-\mathbf{q}_{\alpha} \mathbf{q}_{\beta} \frac{c^{2}}{\omega^{2}+c^{2} \mathbf{q}^{2}}\right) .
\end{aligned}
$$

One immediately confirms current conservation, i.e.

$$
q_{\alpha} q_{\beta} G_{; \alpha \beta}(q)=\omega^{2} G_{; 00}(q) .
$$

These results show the overall consistency of the hydrodynamic approach.

\section{Perturbative corrections}

Up till now we only considered the quadratic part of the hydrodynamic action given by Eq. (11). In order to calculate perturbative corrections to the propagators given by Eq. (17) we now consider the cubic part of the action. This leads to the phonon decay processes, i.e. a finite phonon life-time, as well as a second sound branch (See, e. g., Ref. [12]).

The diagrams corresponding to the corrections for Green's functions in the second order perturbation theory are shown in Fig. 2. 


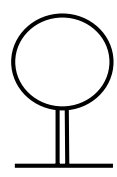

$a$

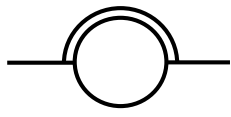

$b$

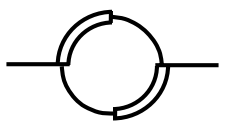

$c$

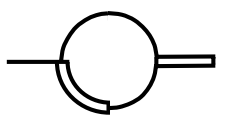

$d$

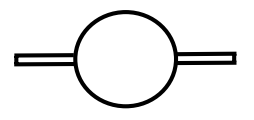

$e$

Fig. 2. Self-energy corrections to hydrodynamical propagators given in Eq. (17). Graphs (a), (b) and (c) correspond to $\Sigma_{\varphi \varphi}$, graph (d) to the $\Sigma_{\varphi \pi}$ and graph (e) to $\Sigma_{\pi \pi}$.

All diagrams except (a) will provide finite corrections. Diagram (a) equals zero since the intermediate $g_{\pi \pi}$ propagator which connects the external propagator lines to the loop carries zero momentum and energy. It is possible to construct other diagrams of the type (a), however they all vanish.

We calculate the imaginary part of the spectrum at $T=0$ using the unperturbed Green's functions $G_{0}(17)$ according to

$$
\operatorname{det} G^{-1}(q)=\operatorname{det}\left(G_{0}^{-1}(q)-\Sigma(q)\right)=0 .
$$

For explicit calculations it is more convenient to use the unperturbed Green's function in the form of Eq. (12). After analytical continuation, $i \omega \rightarrow E$, Eq. (40) takes (in the limit $\mathbf{q} \rightarrow 0$ ) the form of a quadratic equation

$$
E^{2}\left[1+\Sigma_{\pi \pi}(E) m c^{2} \frac{p_{\mu \mu}}{p_{n_{0} n_{0}} p_{\mu}}\right]-2 i E \Sigma_{\varphi \pi}(E) \frac{m c^{2}}{n} \frac{p_{\mu n_{0}}}{p_{n_{0} n_{0}}}-c^{2} \mathbf{q}^{2}\left[1-\Sigma_{\pi \pi}(E) \frac{1}{p_{n_{0} n_{0}}}+\Sigma_{\varphi \varphi}(E) \frac{m c^{2}}{p_{\mu} \mathbf{q}^{2}}\right]=0 .
$$

It is not possible to solve this equation exactly, therefore we have to look for an approximate solution assuming that $\Sigma$ is small for small energy and momentum. This assumption will be justified a posteriori. To this end we expand $\Sigma(E)$ around the unperturbed solution $E=\varepsilon(q)=c|\mathbf{q}|$. This allows us, using Eqs. (15) and (16) with $p_{\mu}=n$, to write a solution of this equation in the form

$$
E_{1,2}(\mathbf{q} \rightarrow 0)=-i \frac{m c^{2}}{n} \frac{d n_{0}}{d \mu} \Sigma_{\varphi \pi}(\varepsilon(\mathbf{q})) \pm c|\mathbf{q}|\left[1-\frac{1}{2} \Sigma_{\pi \pi}(\varepsilon(\mathbf{q})) \frac{m c^{2}}{n}\left(\frac{d n_{0}}{d \mu}\right)^{2}-\frac{1}{2} \Sigma_{\varphi \varphi}(\varepsilon(\mathbf{q})) \frac{m}{n} \frac{1}{\mathbf{q}^{2}}\right] .
$$

The imaginary part of Eq. (42) is given by the following expression

$$
\begin{aligned}
\operatorname{Im} E_{1,2}(\mathbf{q} \rightarrow 0) & =\Delta(\mathbf{q} \rightarrow 0)=-\frac{m c^{2}}{n} \frac{d n_{0}}{d \mu} \operatorname{Re} \Sigma_{\varphi \pi}(\varepsilon(\mathbf{q})) \\
& \mp c|\mathbf{q}|\left[\frac{m c^{2}}{2 n}\left(\frac{d n_{0}}{d \mu}\right)^{2} \operatorname{Im} \Sigma_{\pi \pi}(\varepsilon(\mathbf{q}))+\frac{m}{2 n} \frac{1}{\mathbf{q}^{2}} \operatorname{Im} \Sigma_{\varphi \varphi}(\varepsilon(\mathbf{q}))\right] .
\end{aligned}
$$

The expressions for the self-energies $\Sigma_{\varphi \pi}(i \omega), \Sigma_{\varphi \varphi}(i \omega)$ and $\Sigma_{\pi \pi}(i \omega)$ are obtained from the corresponding graphs on Fig. 2 using the Feynman rules. According to these rules each internal line corresponds to a free propagator of (17), and for small momenta we may neglect the constant terms $\sim 1 / p_{n_{0} n_{0}}$. One must integrate over all internal momenta and sum over all internal Matsubara frequencies. As an example, the full expression for $\Sigma_{\varphi \pi}$ is given by

$$
\Sigma_{\varphi \pi}(i \omega, \mathbf{q})=\frac{T}{(2 \pi)^{3} m^{2}} \int d^{3} q_{2} d^{3} q_{3}\left(\mathbf{q} \cdot \mathbf{q}_{3}\right)\left(\mathbf{q}_{2} \cdot \mathbf{q}_{3}\right) \sum_{\omega_{2}, \omega_{3}} \eta\left(q, q_{2}, q_{3}\right) g_{\varphi \varphi}\left(\mathbf{q}_{2}, i \omega_{2}\right) g_{\pi \varphi}\left(\mathbf{q}_{3}, i \omega_{3}\right)
$$

with

$$
\eta\left(q, q_{2}, q_{3}\right) \equiv \delta\left(\mathbf{q} \pm \mathbf{q}_{2} \mp \mathbf{q}_{3}\right) \delta_{\omega \pm \omega_{2}, \pm \omega_{3}}+\delta\left(\mathbf{q}-\mathbf{q}_{2}-\mathbf{q}_{3}\right) \delta_{\omega-\omega_{2}, \omega_{3}} .
$$

Note, that $\eta$ contains three summands, the first term corresponding to the upper signs and the second to the lower signs. The Matsubara sum is executed by an integration in the complex plane over the contour shown in Fig. 3 by virtue of the residue theorem

$$
\sum_{n=-\infty}^{\infty} f\left(i \omega_{n}\right)=\frac{1}{2 \pi i} \int_{c} d z n_{B}(z) f(z), \quad n_{B}(z)=\left(e^{\beta z}-1\right)^{-1} .
$$

Here, $f(z)$ corresponds to the product of the unperturbed Green's functions in Eq. (44). 


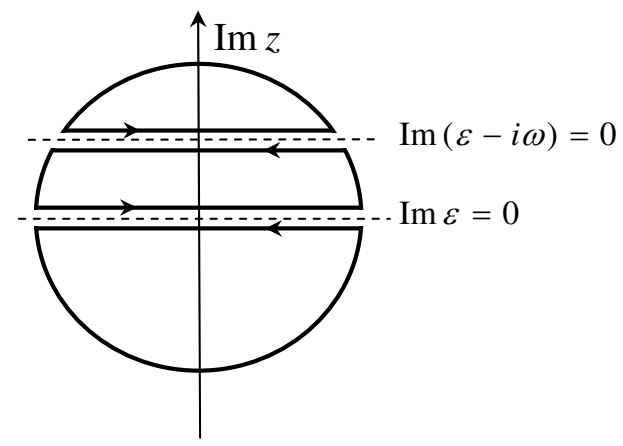

Fig. 3. The contour of integration in the complex $z$-plane.

After the contour integration and summation over all possible directions of the 4-momenta in the diagrams one finds expressions for the real and imaginary parts of the self-energies at $\omega=\varepsilon(\mathbf{q})$

$$
\begin{aligned}
\operatorname{Im} \Sigma_{\varphi \varphi}(\varepsilon(\mathbf{q})) & =\frac{c^{2}\left(1-e^{-\beta \varepsilon(\mathbf{q})}\right)}{32 \pi^{2} n^{2}}\left(\frac{d n_{0}}{d \mu}\right)^{2} \int d^{3} q_{2} d^{3} q_{3} \varepsilon_{2} \varepsilon_{3} n_{2} n_{3} 3 \frac{\left(\mathbf{q} \cdot \mathbf{q}_{3}\right)^{2}}{\mathbf{q}_{3}^{2}}\left[2 \delta(1+2-3) e^{\beta \varepsilon_{3}}+\delta(1-2-3) e^{\beta \varepsilon}\right](47) \\
& +\frac{\left(\mathbf{q} \cdot \mathbf{q}_{3}\right)\left(\mathbf{q}_{2} \cdot \mathbf{q}_{3}\right)}{\left|\mathbf{q}_{2}\right|\left|\mathbf{q}_{3}\right|}\left[2 \delta(1+2-3) e^{-\beta \varepsilon_{3}}-\delta(1-2-3) e^{\beta \varepsilon(\mathbf{q})}\right], \\
\operatorname{Im} \Sigma_{\pi \pi}(\varepsilon(\mathbf{q})) & =\frac{c^{2}\left(1-e^{-\beta \varepsilon(\mathbf{q})}\right)}{32 \pi^{2} n^{2}} \int d^{3} q_{2} d^{3} q_{3} \varepsilon_{2} \varepsilon_{3} n_{2} n_{3} 3 \frac{\left(\mathbf{q}_{2} \cdot \mathbf{q}_{3}\right)}{\mathbf{q}_{2}^{2} \mathbf{q}_{3}^{2}}\left[2 \delta(1+2-3) e^{\beta \varepsilon_{3}}+\delta(1-2-3) e^{\beta \varepsilon_{1}}\right], \\
\operatorname{Re} \Sigma_{\varphi \pi}(\varepsilon(\mathbf{q})) & =\frac{\left(1-e^{-\beta \varepsilon(\mathbf{q})}\right)}{32 \pi^{2} n^{2}} \frac{d n_{0}}{d \mu} \int d^{3} q_{2} d^{3} q_{3} \varepsilon_{1} \varepsilon_{2} \varepsilon_{3} n_{2} n_{3} \frac{\left(\mathbf{q} \cdot \mathbf{q}_{3}\right)\left(\mathbf{q}_{2} \cdot \mathbf{q}_{3}\right)}{|\mathbf{q}|\left|\mathbf{q}_{2}\right| \mathbf{q}_{3}^{2}}\left[2 \delta(1+2-3) e^{\beta \varepsilon_{3}}+\delta(1-2-3) e^{\beta \varepsilon_{1}}\right],
\end{aligned}
$$

with

$$
\begin{aligned}
& \delta(1 \pm 2-3) \equiv \delta\left(\mathbf{q} \pm \mathbf{q}_{2}-\mathbf{q}_{3}\right) \delta\left(\varepsilon \pm \varepsilon_{2}-\varepsilon_{3}\right) \\
& n_{i} \equiv\left(e^{\beta \varepsilon_{i}}-1\right)^{-1}, \quad \varepsilon_{i}=c\left|\mathbf{q}_{i}\right|
\end{aligned}
$$

In the above equations we assume that $\left(\mathbf{q}_{i} \cdot \mathbf{q}_{j}\right) /\left|\mathbf{q}_{i}\right|\left|\mathbf{q}_{j}\right| \sim 1$ at small momenta. The angle $\theta$ between the momenta of two particles is small in this limit, and we may take $\cos \theta=1$. Using this approximation we are now able to find the imaginary part of the spectrum. Using Eqs. (43) and (47) we find

$$
\Delta(\mathbf{q})=\frac{9 m c^{2}\left(1-e^{-\beta \varepsilon(\mathbf{q})}\right)}{128 \pi^{2} n^{3}}\left(\frac{d n_{0}}{d \mu}\right)^{2} \int d q_{2}^{3} d q_{3}^{3} \varepsilon_{1} \varepsilon_{2} \varepsilon_{3} n_{2} n_{3}\left[2 \delta(1+2-3) e^{\beta \varepsilon_{3}}+\delta(1-2-3) e^{\beta \varepsilon_{1}}\right] .
$$

In the low density limit this result coincides with the expression obtained by Popov [12], which at zero temperature $(\beta \rightarrow \infty)$ corresponds to Belyaev's result [5]

$$
\Delta(\mathbf{q})=\frac{3 \mathbf{q}^{5}}{640 \pi \rho m}
$$

\section{DISCUSSION AND CONCLUSIONS}

The determination of the infrared response of a strongly interacting Bose system from the microscopic field theory is plagued by infrared divergences of various integrals. This calls for a systematic renormalization, which has been performed recently in Ref. [15]. The essential results of this procedure are equivalent to Eqs. (24) obtained using Popov's hydrodynamic approach. This shows that Popov's approach correctly identifies the infrared fixed point of the renormalized effective action.

The transverse correlation function shows a "physical" divergence for $\mathbf{q}, \omega \rightarrow 0$, which is due to a Goldstone mode related to massless phase fluctuations. The logarithmic divergence exhibited by the longitudinal correlation function in a long wavelength limit may be unexpected at first since the longitudinal correlations are determined primarily by a massive mode. This logarithmic divergence stems from phase fluctuations as well, which contribute to the longitudinal response in the second order. 


\section{A. CHUMACHENKO, S. VILCHYNSKYY, M. WEYRAUCH}

These features can all be understood physically using the picture of symmetry breaking as discussed by Patashinskii and Pokrovskii [17] in an analogous magnetic system. Technically, the observed divergences of the correlation functions are directly related to the vanishing of $\Sigma_{12}$ at zero momentum and energy. More precisely, $\Sigma_{12}$ vanishes non-analytically with an infinite slope at zero momentum. This is a very important fact, which precludes an expansion of the correlation functions around $\mathbf{q}=0$.

We apply the hydrodynamic formalism in order to find expressions for the density-density response as well as the density-current response, which are infrared finite. We also calculate the quasi-particle density for $T \ll c|\mathbf{q}|$ and $T \gg c|\mathbf{q}|$. Well-established results are confirmed.

The hydrodynamic action proposed by Popov contains a cubic term in the phonon fields. This term, which resembles the residual interaction of the quasi-particles, describes a quasi-particle decay. In this paper we used this residual interaction in order to calculate the intrinsic width of the phonon response in the second order perturbation theory. In the low density limit our result agrees with Popov's result given in Ref. [12].

The parameters of the hydrodynamic action are related to the bare coupling constants through the renormalization flow. The renormalization group analysis shows that the bare action flows into a unique fixed point, the parameters of which are given by macroscopic observables like the speed of first sound or compressibility. How- ever, no quantitative relation between the bare interaction and the renormalized action and its parameters is established.

At larger momenta and energies even the structure of the response depends on the details of the interaction. Phenomenologically it is described by the maxon-roton response, and it is a real challenge to relate this response to the microscopic action. To our best knowledge a convincing quantitative connection between the observed response and the microscopic action at larger energy and momentum transfer has only been achieved using numerical methods, e. g. quantum Monte Carlo [18].

Field theoretical methods have been used in order to explain the maxon-roton spectrum [25-27], but these attempts do not seem to be fully convincing. For instance, the Nepomnyashchii-Pashitskii approach [25, 26] does not implement gauge symmetry breaking in a satisfactory way so that e.g. $\Sigma_{12}(0)$ is predicted to be finite. It is a major challenge to reformulate this and similar approaches in such a way that the gauge symmetry breaking is built in satisfactorily.

\section{Acknowledgments}

S. V. would like to thank the Deutsche Forschungsgemeinschaft for support. S. V. and A. C. express their gratitude to Physikalisch-Technische Bundesanstalt for support and hospitality. The authors thank M. Reginatto for useful discussions.
[1] L. D. Landau, Zh. Eksp. Teor. Fiz. 11, 592 (1941); J. Phys. USSR 5, 71 (1941).

[2] L. D. Landau, Zh. Eksp. Teor. Fiz. 17, 91 (1947); J. Phys. USSR 11, 91 (1947).

[3] N. N. Bogolubov, J. Phys. USSR 11, 23 (1947).

[4] S. T. Belyaev, Zh. Eksp. Teor. Fiz. 34, 417 (1958); Sov. Phys. JETP 34, 289 (1958).

[5] S. T. Belyaev, Zh. Eksp. Teor. Fiz. 34(2), 433 (1958); Sov. Phys. JETP 34, 299 (1958).

[6] N. M. Hugenholtz, D. Pines, Phys. Rev. 116, 489 (1959).

[7] J. Gavoret, P. Nozières, Ann. Phys. 28, 349 (1964).

[8] P. C. Hohenberg, P. C. Martin, Ann. Phys. 34, 291 (1965).

[9] P. W. Anderson, Basic notions of condensed matter physics (Westview Press, 1997).

[10] A. A. Abrikosov, L. P. Gorkov, I. E. Dzyaloshinski, Methods of Quantum Field Theory in Statistical Physics (Prentice-Hall. Englewood Clifts N. J., Princeton, New Jersey, 1963).

[11] Yu. A. Nepomnyashchii, A. A. Nepomnyashchii, Zh. Eksp. Teor. Fiz. 75, 976 (1978); Sov. Phys. JETP 48, 493 (1978).

[12] V. N. Popov, Functional Integrals in Quantum Field Theory and Statistical Physics (D. Reidel Publishing Company, Dordrecht, 1983).

[13] V. N. Popov, Functional Integrals and Collective Excita- tions (Cambridge University Press, Cambridge, 1987).

[14] V. N. Popov, A. V. Serednyakov, Zh. Eksp. Teor. Fiz. 77, 377 (1979); Sov. Phys. JETP 50, 193 (1979).

[15] F. Pistolesi, C. Castellani, C. Di Castro, G. C. Strinati, Phys. Rev. B 69, 024513 (2004).

[16] C. Castellani, C. Di Castro, F. Pistolesi, G. C. Strinati, Phys. Rev. Lett. 78, 1612 (1997).

[17] A. Z. Patashinskii, V. L. Pokrovskii, Fluctuation Theory of Phase Transitions (Pergamon Press, Oxford, 1979).

[18] D. M. Ceperley, Rev. Mod. Phys. 67, 279 (1951).

[19] F. J. Dyson, Phys. Rev. 75, 1763 (1949).

[20] T. Davison, E. Feenberg, Phys. Rev. 171, 221 (1968).

[21] I. O. Vakarchuk, J. Phys. Stud. 8, 223 (2004).

[22] John W. Negele, Henri Orland, Quantum Many-Particle Systems. (Perseus Books Publishing, Reading, Mass., 1998).

[23] Yu. A. Nepomnyashchii, Phys. Rev. B 46, 6611 (1992).

[24] S. Georgini, L. Pitaevskii, S. Stringari, Phys. Rev. B 46, 6374 (1992).

[25] Yu. A. Nepomnyashchii, E. A. Pashitskii, Zh. Eksp. Teor. Fiz. 98, 178 (1990); Sov. Phys. JETP 71, 98 (1990).

[26] E. A. Pashitskii, S. V. Mashkevich, S. I. Vilchynskyy, Phys. Rev. Lett. 89, 075301 (2002).

[27] J. Szwabinski, M. Weyrauch, Phys. Rev. B 64, 184512 (2001). 


\title{
ІНФРАЧЕРВОНА ПОВЕДІНКА ВІДГУКУ ДЛЯ СИЛЬНОВЗАЕМОДІЮЧИХ БОЗЕ-СИСТЕМ
}

\author{
А. Чумаченко ${ }^{1}$, С. Вільчинський ${ }^{1}$, М. Вейраух ${ }^{2}$ \\ ${ }^{1}$ Київсъкий національний університет імені Тараса Шевченка, Украӥна \\ ${ }^{2}$ Фізико-технічний федеральний інститут, \\ D-38116, Брауншвай', Федеративна Республіка Німеччина
}

\begin{abstract}
Обговорено теоретико-польові основи квазічастинкового підходу Ландау для сильновзаємодіючих бозонів. Коротко висвітлено історичний розвиток мікроскопічної теорії, починаючи з польової теорії Бєляєва й закінчуючи сучасною теорією ренорм-групи, особливу увагу приділено проблемі інфрачервоних розбіжностей. Показано докладно, що кореляційні функції, які отримав за допомогою методу функціонального інтегрування Попов на основі гідродинамічної дії, збігаються з останніми результатами теорії ренорм-групи. Також обговорено розпад фононів.
\end{abstract}

